\title{
Color Image Compression using PCA
}

\author{
Mohammad Mofarreh-Bonab \\ Department of Electrical Engineering, University of \\ Bonab, Bonab 5551761167, Iran
}

\begin{abstract}
Principal Component Analysis (PCA) is an efficient method for compressing high dimensional databases [1]. For image compression, it is called Hotelling or KL transform. The central idea of PCA is to reduce the dimensionality of a data set in which there are a large number of interrelated variables. [2] This reduction is achieved by transforming to a new set of variables, the principal components, which are uncorrelated, and which are ordered so that the first few retain most of the variation present in all of the original variables. Computation of the principal components reduces to the solution of an Eigen value - Eigen vector problem for a positive-semi definite symmetric matrix [2]. In spite of ordinary applications which utilize the PCA method for dataset compression, in this paper, a new method is introduced to compress a single image in RGB color space using the correlations between three Red, Green and Blue color domains.
\end{abstract}

\section{General Terms}

Color image compression, Principal Component Analysis, PCA, RGB

\section{Keywords}

Hotelling, compression ratio, Eigen value, Eigen vector, Principal Component Analysis, color image compression

\section{INTRODUCTION}

PCA method utilizes the correlations between the elements of a dataset to decrease the dimensionality of it, so, the PCA originally is applicable to a dataset rather than a single image. To utilize PCA for compressing a single color image, the fact that there are high correlations between each color domains of an image is focused in this paper. In other words, the color components of an image could be supposed as separated images, so applying the PCA can help to compress the image. In this paper, a new method for compressing color images is introduced that utilizes the PCA method.

\section{PCA METHOD FOR IMAGES [4]}

A grayscale square image can be expressed with $\mathrm{N} \times \mathrm{N}$ pixels which form a $1 \times \mathrm{N}^{2}$ vector. For $\mathrm{M}$ images, a $\mathrm{M} \times \mathrm{N}^{2}$ matrix can be formed as (1):

$X=\left[\begin{array}{c}F_{1} \\ \vdots \\ F_{M}\end{array}\right], \begin{gathered}F_{1}=\left(x_{11}, x_{12}, \ldots, x_{1 N^{2}}\right) \\ F_{M}=\left(x_{M 1}, x_{M 2}, \ldots, x_{M N}\right)\end{gathered}$

So, the mean vector $\bar{M}_{x}$ and the mean matrix $\widetilde{M}_{x}$ and the covariance matrix $\mathrm{C}_{\mathrm{x}}$ can be defined [1], [5];

$$
\begin{gathered}
\bar{M}_{x}=\frac{1}{M}\left[\begin{array}{c}
\sum_{k=1}^{N^{2}} x_{1, k} \\
\sum_{k=1}^{N^{2}} x_{2, k} \\
\cdot \\
\cdot \\
\sum_{k=1}^{N^{2}} x_{M, k}
\end{array}\right]_{M \times 1}=\left[\begin{array}{c}
m_{1} \\
m_{2} \\
\cdot \\
\cdot \\
\cdot \\
m_{M}
\end{array}\right], \\
\widetilde{M}_{x}=\left[\bar{M}_{x}, \bar{M}_{x}, \bar{M}_{x}, \ldots, \bar{M}_{x}\right]_{M \times N^{2}}
\end{gathered}
$$

\author{
Mostafa Mofarreh-Bonab \\ Department of Electrical and Computer \\ Engineering, Shahid Beheshti University, Tehran \\ 1983963113, Iran,
}

$C_{x}=\left[c_{i, j}\right]_{M \times M} c_{i, j}=\frac{1}{N^{2}-1} \times \sum_{k=1}^{N^{2}}\left[\left(x_{i, k}-\bar{M}_{x}(i, 1)\right) \times\right.$
$\left.\left(x_{j, k}-\bar{M}_{x}(j, 1)\right)\right]$

The values of $C_{x}$ matrix are real values, so $M$ Eigen vectors $v_{i}$ and $M$ Eigen values $\lambda_{\mathrm{i}}$ can be obtained [6].

$\forall i \in\{1,2, \ldots, M\} \quad C_{x} \cdot v_{i}=\lambda_{i} \cdot v_{i}$

Each Eigen vector which its Eigen value is greater has more information [7]. If the Eigen vectors be sorted in descending order, the matrix $\mathrm{V}$ can be defined as below:

$V=\left[v_{1}, v_{2}, \ldots, v_{M}\right]_{M \times M}^{T}$

That

$$
\begin{aligned}
& V_{i}=\left[\begin{array}{c}
V_{i}(1) \\
V_{i}(2) \\
\cdot \\
\cdot \\
V_{i}(M)
\end{array}\right] \Rightarrow V=\left[\begin{array}{c}
V_{1}(1), \ldots, V_{M}(1) \\
V_{1}(2), \ldots, V_{M}(2) \\
\cdot \\
\cdot \\
V_{1}(M), \ldots, V_{M}(M)
\end{array}\right]_{M \times M}^{T}= \\
& {\left[\begin{array}{c}
V_{1}(1), \ldots, V_{1}(M) \\
V_{2}(1), \ldots, V_{2}(M) \\
\cdot \\
\cdot \\
V_{M}(1), \ldots, V_{M}(M)
\end{array}\right]_{M \times M}}
\end{aligned}
$$

The first row of the matrix $V$ is the Eigen vector that its Eigen value is the largest between the Eigen values. Now the Hotelling transform can be applied:

$$
\begin{aligned}
& Y=V \cdot\left(X-\widetilde{M}_{x}\right) \Rightarrow \\
& X=V^{-1} \cdot Y+\widetilde{M}_{x}=V^{T} . Y+\widetilde{M}_{x} \\
& X=\widetilde{M}_{x}+\left[\begin{array}{c}
V_{1}(1), \ldots, V_{1}(M) \\
V_{2}(1), \ldots, V_{2}(M) \\
\cdot \\
\cdot \\
V_{M}(1), \ldots, V_{M}(M)
\end{array}\right]_{M \times M} \quad\left[\begin{array}{c}
Y(1,1), \ldots, Y\left(1, N^{2}\right) \\
Y(2,1), \ldots, Y\left(2, N^{2}\right) \\
\cdot \\
\cdot \\
Y(M, 1), \ldots, Y\left(M, N^{2}\right)
\end{array}\right]_{M \times N^{2}}
\end{aligned}
$$$$
X(i, j)=\sum_{p=1}^{M}\left[v_{i}(p) Y(p, j)\right]+m_{i}
$$

Processing Eigen values and Eigen vectors, if images of database have more correlation with each other, differences between Eigen values are significant. In fact the Eigen vectors with the highest Eigen values are the principal components of the database [8]. Having all components of database in descending order of significance, the fewer significant components can be ignored in order to compress the database. Considering the first $\mathrm{k}$ Eigen vectors from the $\mathrm{M}$ Eigen vectors $(\mathrm{K}<\mathrm{M})$, the $\widehat{\mathrm{X}}$ matrix that is slightly different with $\mathrm{X}$ matrix can be retrieved. The values of $\widehat{X}$ matrix are near to values of $\mathrm{X}$ matrix, so:

$$
\begin{aligned}
& V_{k}= {\left[v_{1}, v_{2}, \ldots, v_{k}\right]_{M \times k}^{T}, k \in\{1,2, \ldots, M\} } \\
& Y_{k}=\left[\begin{array}{c}
Y(1,1), \ldots, Y\left(1, N^{2}\right) \\
Y(2,1), \ldots, Y\left(2, N^{2}\right) \\
\cdot \\
\cdot \\
Y(k, 1), \ldots, Y\left(k, N^{2}\right)
\end{array}\right]_{k \times N^{2}}
\end{aligned}
$$


$\hat{X}=V_{k}{ }^{-1} \cdot Y+\widetilde{M}_{x}=V_{k}{ }^{T} \cdot Y+\widetilde{M}_{x}$

The $\widehat{\mathrm{X}}$ matrix is compressed matrix which obtained from $\mathrm{X}$ matrix. So the compression ratio that is the ratio of the required memory to save $\widehat{X}$ to the required memory to save $\mathrm{X}$ can be calculated as (9) and (10):

Memory ratio $=\frac{\text { required memory to save } \hat{X}}{\text { required memory to save } X} \triangleq \frac{m e m 1}{m e m 2}$

mem $1=$ required memory to save $\left(V_{k}{ }^{T}, Y_{k}, \widetilde{M}_{x}\right)$

$\Rightarrow$ Memory ratio $\left.\right|_{\text {original HOTTELING }}=\frac{k M+k N^{2}+M}{M N^{2}}$

\section{EXTENDED PCA FOR SINGLE COLOR IMAGE COMPRESSION}

In [3], a PCA based method is introduced to compress a grayscale image. This method divides the image into several bands and the rows in each band are supposed as separated images. Taking advantage of similarities between the rows in each band, the PCA is applied to compress the image [3]. In the new method which will be explained in more details in this paper, the color components of a color image is extracted and the method that is mentioned in [3] is applied to each of them, but this time, instead the row similarities, the correlations between color components of each band are utilized for compression.

A color image in RGB space consists of three color domains, the Red, Green and Blue domains. Figure 1 shows the Lena image and its $\mathrm{R}, \mathrm{G}$ and $\mathrm{B}$ components.
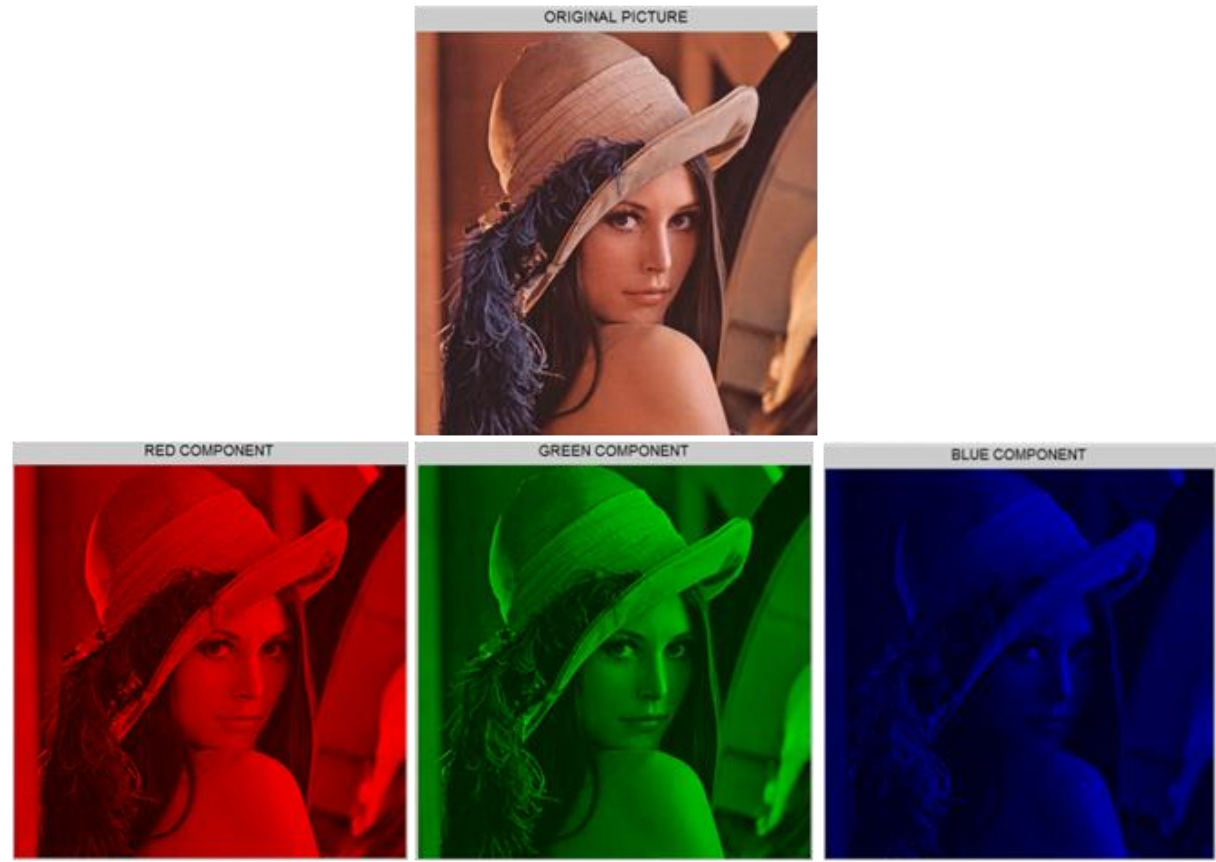

Fig 1: Lena and its RGB color components

Three color components have high correlations. For example, from histogram point of view, figure 2 shows the histograms of three color components of Lena image.

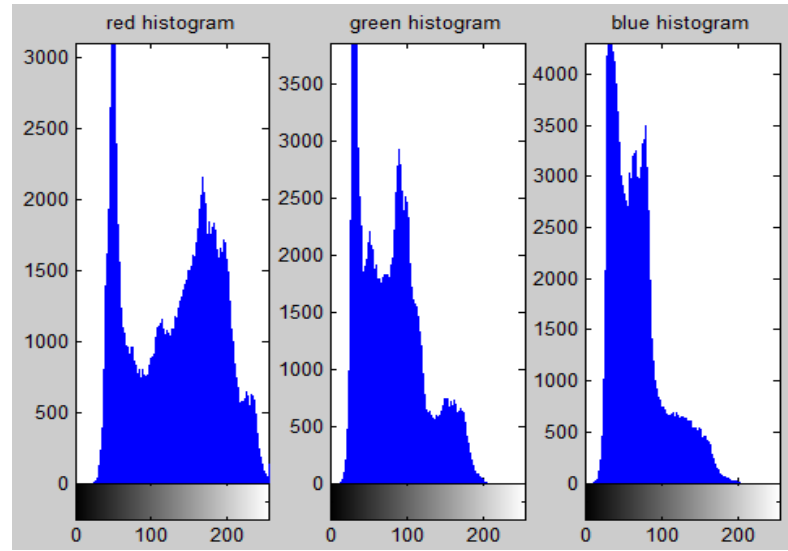

Figure 2: the Histograms of Red, Green and Blue components of Lena
Figure 2 shows that there are strong similarities between the color components, especially between Green and Blue. In the following, each of these components is divided into several bands with equal widths and then, the introduced method in [3] is applied to these bands as follows.

In this paper, each color component is divided into 16 horizontal bands and the matrix $\mathrm{X}_{\mathrm{i}}$ which will be the $\mathrm{i}^{\text {th }}$ input of Hotteling transform is produced. In the other words, the $i^{\text {th }}$ row of $\mathrm{X}$ matrix contains the brightness values of the image that is produced by putting three color bands of original image together.

Figure 3 shows the input of Hotteling transform for $8^{\text {th }}$ band.

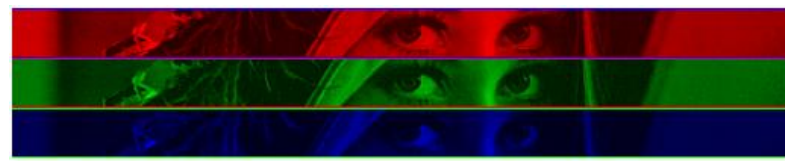

Figure 3: The input for Hotteling transform for $8^{\text {th }}$ band 


\section{SIMULATION RESULTS}

Simulation results show that high compression ratios can be obtained by using of mentioned method. For example, the results of applying this method to the Lena image is shown in figure 4. Notice to the compression parameters - SSIM [9] and compression Ratio- that are shown at the bottom of each result. In figures 5 and 6, SSIM values vs. the percentage of compression ratios are plotted for Lenna and Mandrill images respectively.
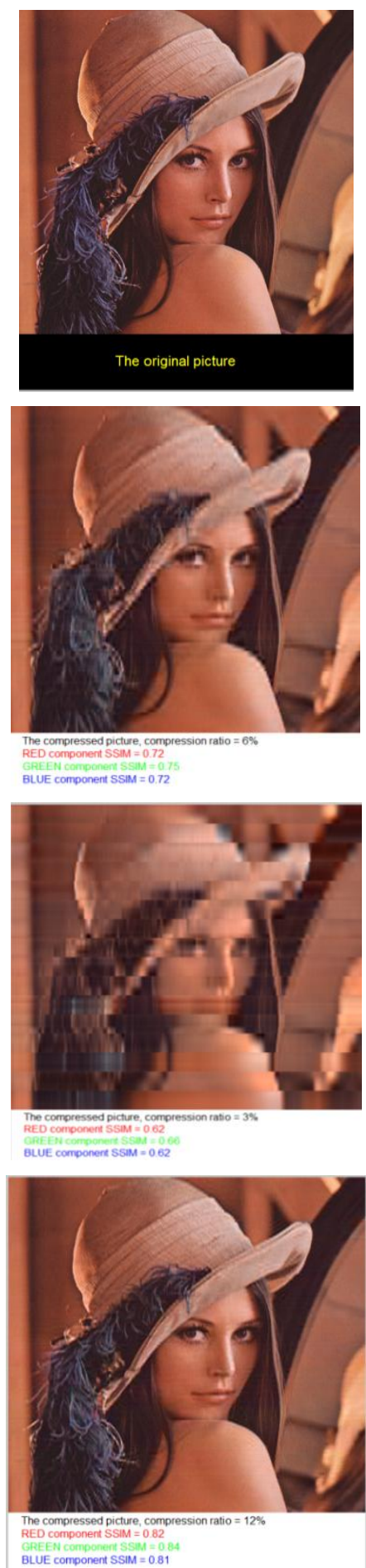

Figure 4: SSIM and compression Ratio for each component of Lena using mentioned method

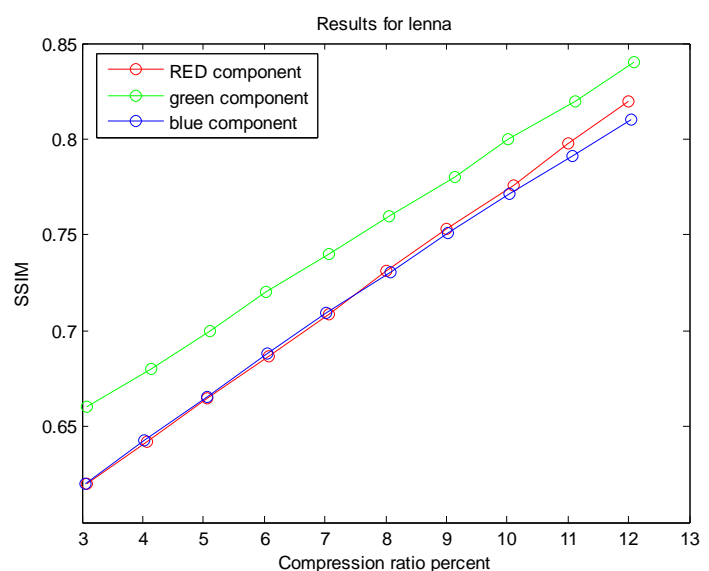

Figure 5: SSIM values vs. the percentage of compression ratio for Lenna image

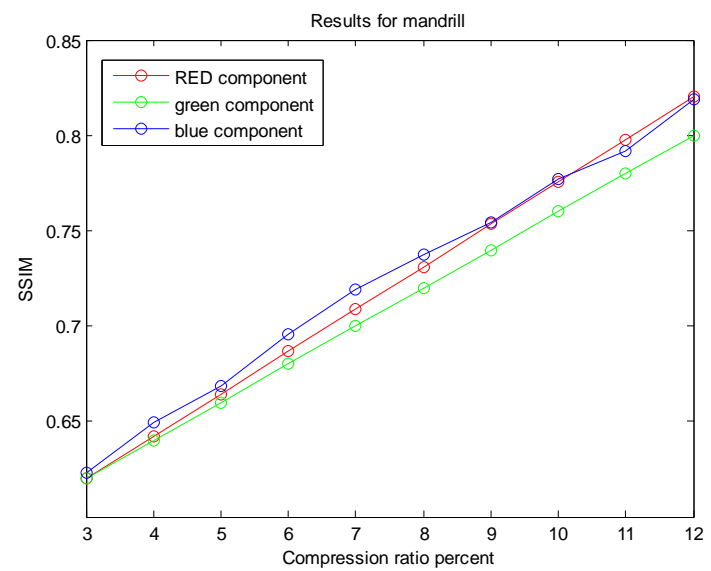

Figure 6: SSIM values vs. the percentage of compression ratio for Mandrill image

\section{CONCLUSIONS}

In this paper, a new PCA based method to compress color images is introduced which can be utilized to compress single image rather than a set of separated images. This method uses the correlations between three color components of an image. This method can be utilized in parallel mode to increase the compression speed. Furthermore, a more precise method for selecting the bands of image can be utilized to improve the performance of compression.

\section{REFERENCES}

[1] R. Gonzales and R. Woods, Digital Image Processing, Prentice-Hall, 3rd Ed., 2008. ISBN number 9780131687288 .

[2] I.T. Jolliffe, Principal Component Analysis, Springer, $2^{\text {nd }}$ edition, 2002, XXIX, 487 p. 28 illus.

[3] M. Mofarreh-Bonab, M. Mofarreh-Bonab, "A New Technique for Image Compression Using PCA", International Journal of Computer Science \& Communication Networks IJCSCN (2249-5789), Vol. 2(1), 111-116, 2012

[4] M. Mofarreh-Bonab, M. Mofarreh-Bonab, "FACE DATABASE COMPRESSION BY HOTELLING TRANSFORM USING A NEW METHOD”, 2nd World Conference on Information Technology (WCIT - 2011), Antalya, Turkey, 23-27 November 2011. 
[5] A.M. Aznaveh, F. Torkamani Azar, A. Mansouri, "FACE DATA BASE COMPRESSION BY HOTELLING TRANSFORM USING SEGMENTATION", Signal Processing and Its Applications, 2007. ISSPA 2007.

[6] Li. Xuelong, Y. Pang, "L1-Norm-Based 2DPCA", IEEE TRANSACTIONS ON SYSTEMS, MAN, AND CYBERNETICS-PART B: CYBERNETICS, VOL. 40, No. 4, AUGUST 2009.
[7] P.K. Pandey, Y. Singh, S. Tripathi, "Image Processing using Principle Component Analysis", International Journal of Computer Applications IJCA (0975 - 8887) Volume 15- No.4, February 2011.

[8] A.A. Mohammed, R. Minhas, Q.M. Jonathan Wu, M.A. Sid-Ahmed, "Human face recognition based on multidimensional PCA and extreme learning machine", Pattern Recognition, Volume 44, Issues 10-11, OctoberNovember 2011, Pages 2588-2597.

[9] Z. Wang, A. C. Bovik, H. R. Sheikh and E. P. Simoncelli, "Image quality assessment: From error visibility to structural similarity", IEEE Transactions on Image Processing, vol. 13, no. 4, pp. 600-612, Apr. 2004 